\title{
BRAIN FUNCTION IN DUCHENNE MUSCULAR DYSTROPHY
}

The role of dystrophin disorders in the CNS function of boys with Duchenne muscular dystrophy (DMD) and the dystrophin-deficient mdx mouse, an animal model of DMD, is reviewed at the University of New South Wales, University of Sydney, Australia. DMD boys have a lower IQ (average 85) than normal. The degree of cognitive impairment may be associated with a mutation in the dystrophin isoform, Dp71. Brain histology shows abnormalities in dendrites and loss of neurones, and biochemical changes are indicative of CNS pathology, including an increase in levels of choline-containing compounds. Studies in the mdx mouse have shown a disruption of GABA receptors, that may impact on the action of certain drugs, sleep disorders, and motor control in DMD. Biochemical lesions are particularly prominent in the cerebellum and hippocampus, regions involved in learning disabilities, especially dyslexia and verbal memory deficits. EEG studies have shown a higher proportion of abnormalities in DMD than normal subjects, although many have lacked adequate controls. A reduction in motor cortex excitability, measured by transcranial magnetic stimulation, is attributed to an aberrant synaptic functioning. (Anderson JL, Head SI, Rae C, Morley JW. Brain function in Duchenne muscular dystrophy. Brain Jan 2002;125:4-13). (Respond: Professor JW Morley, School of Physiology and Pharmacology, University of New South Wales, Sydney 2052, Australia).

COMMENT. Duchenne muscular dystrophy, an X-linked inherited disease affecting 1 in 3300 live male births, is caused by the mutation of the dystrophin gene. The dystrophin protein is found in skeletal muscle and also in the CNS. The role of dystrophin and its absence or disruption in the CNS are significant in relation to the management and education of DMD patients. In addition to muscular weakness, and cognitive and learning disabilities, children with DMD have problems in motor control that may be partially attributed to CNS pathology. From a clinical standpoint, a Babinski response, a not infrequent finding in DMD, should not be interpreted as a pseudo sign due to muscle weakness and imbalance.

\section{ELEVATED CK LEVELS IN NEWBORN AND MATERNAL POLYMYOSITIS}

Two infants born to mothers with pregnancy-related polymyositis (PM) had unusually elevated serum creatine kinase (CK) levels, in a report from the University of Milan, Italy. In patient 1 , mother had T cell-mediated PM and CK levels of $2080 \mathrm{U} / \mathrm{L}$ at 1 week before cesarean delivery, and $>8000 \mathrm{U} / \mathrm{L}$ for 3 months after delivery. The infant's CK was 2000 at birth, 4000 at 45 days, and normal $(<185)$ at 4 months; breast feeding was continued for 2 months. Neurologic exam was normal in the infant, but showed increasing proximal weakness in the mother. Maternal recovery following steroid therapy was accompanied by normal CK levels at 10 months after onset of PM. Patient 2 developed proximal weakness and PM at 6 months in her first pregnancy; her CK levels were 1000-2000 U/L initially and normal after steroid therapy, with recovery by time of delivery. The infant had a normal examination but elevated CK levels (1200 at 10 days, 419 at 1 month, and normal after 2 months). The transplacental passage of maternal autoantibodies to the fetus is considered a possible explanation for the elevated CK in the infants. Chimerism, resulting from the two-way passage of cells between fetal and maternal circulations, is an hypothesis, and transfer of CK during breast feeding is a further possible explanation for the findings. (Messina S, Fagiolari G, Lamperti $\mathrm{C}$ et al. Women with pregnancy-related polymyositis and high serum CK levels in the newborn. Neurology February (1 of 2) 2002;58:482-484). (Reprints: Dr 
M Sciacco, Department of Neurological Sciences, University of Milan, Via F Sforza 35, 20122, Milan, Italy).

COMMENT. Polymyositis during pregnancy is rare and may be associated with stillbirth. In infants who survive, the serum CK may be elevated for extended periods. Normally, CK levels can be increased for 3 to 4 days after birth, especially after vaginal birth.

\section{INFECTIOUS DISORDERS}

\section{INFLUENZA-ASSOCIATED ENCEPHALITIS}

Twenty patients with influenza-associated encephalitis/encephalopathy treated during the 1997-2001 influenza A epidemics in Japan are reported from Niigata City General Hospital, Japan. The mean age was 3 years (range 1 to 12 years). No patient had been inoculated with influenza vaccine. None had received aspirin, but 16 patients had received antipyretics (diclofenac or acetaminophen) before onset of encephalopathy. Most had generalized tonic-clonic seizures, and most were treated with midazolam and corticosteroids intravenously. EEG showed high-voltage slow waves in 9 of 17 patients tested, flat records in 3, and focal discharges in 4 . Five patients died, 8 had neurologic sequelae (mental retardation and epilepsy), and 7 recovered completely. Patients who died had hepatic involvement and disseminated intravascular coagulation; all had antipyretics but none had received acetaminophen alone. Patients who recovered had also received antipyretics. Five who died had acute necrotizing encephalopathy (with symmetric lesions in the thalami, brain stem, and cerebellum), 1 other had hemorrhagic shock and encephalopathy syndrome, and 2 had symptoms resembling Reye's syndrome. In 2 deceased patients, plasma levels of cytokines were very high. Influenza vaccination to protect younger children from encephalitis/encephalopathy is recommended, especially those 5 years of age and under. (Yoshikawa H, Yamazaki S, Watanabe T, Abe T. Study of influenza-associated encephalitis/encephalopathy in children during the 1997 to 2001 influenza seasons. I Child Neurol Dec 2001;16:885-890). (Respond: Dr Hideto Yoshikawa, Department of Pediatrics, Niigata City General Hospital, 2-6-1 Shichikuyama, Niigata 950-8739, Japan).

COMMENT. The incidence of severe acute encephalitis/encephalopathy with influenza has increased in Japanese epidemics in recent years, and $80 \%$ of cases occur in children 0 to 5 years of age. The authors cite 202 cases in the nationwide surveillance, of whom $31 \%$ died, $26 \%$ had residual neurologic sequelae, and $43 \%$ recovered completely. Apart from the lack of vaccination, the reasons for the high incidence, mortality and morbidity were not clear. The above Niigata study shows that acute necrotizing encephalopathy with cerebral edema and thalamic lesions is the reason for the high mortality. The role of antipyretics, especially diclofenac, in the cause of influenza encephalopathy needs further investigation. Hypercytokinemia heralds a poor prognosis. Influenza vaccination is now promoted, especially in young children, and the use of antipyretics is discouraged.

\section{SERIAL MRI IN RASMUSSEN'S ENCEPHALITIS}

The course of Rasmussen's encephalitis (RE) was studied by a correlation of serial MRI and histopathology of surgical specimens in 10 patients ( 7 children, 1 adolescent, 2 young adults) followed at the University of Bonn, Germany, and University of Vienna, Austria. All developed the typical progressive hemispheric 